\title{
ESTUDIO EXPLORATORIO DE LAS CARACTERÍSTICAS SOCIO-DEMOGRÁFICAS Y PSICOPATOLÓGICAS DE PERSONAS DENUNCIADAS COMO AGRESORES DE VIOLENCIA DOMÉSTICA EN LA CIUDAD DE CÓRDOBA.
}

\author{
EXPLORATORY STUDY OF THE SOCIO-DEMOGRAPHIC AND PSYCHOPATHOLOGICAL FEATURES \\ OF PERSONS REPORTED AS PERPETRATORS OF VIOLENCE IN CORDOBA CITY.
}

\author{
Mariela E. Roldan ${ }^{1}$, Mónica Bella ${ }^{1}$, Leandro Dionisio ${ }^{1}$.
}

\section{Resumen:}

La violencia doméstica es actualmente un problema de salud pública debido a su magnitud y repercusiones sociosanitarias. Objetivo: explorar las características epidemiológicas sociodemográficas y psicopatológicas de personas denunciadas como agresores en la ciudad de Córdoba. Material y Métodos: estudio exploratorio, retrospectivo y transversal de los registros de personas denunciadas como presuntos agresores de violencia intrafamiliar en la fiscalía de violencia familiar de los tribunales Judiciales Provinciales de Córdoba Capital en el período junio 2011 a mayo 2012. Resultados: el (86\%) fueron varones y el (14\%) mujeres con una edad de \pm 35 años. La violencia de pareja fue la más prevalente en el (69\%). El grupo etario más comprometido fue el de 20 a 39 años El tipo de lesión más frecuente fue la amenaza (45,6\%), seguida de lesiones graves (35,3\%). La mayor parte de los casos no presentó diagnóstico de trastorno mental $(64,3 \%)$. El trastorno mental más frecuente fue el consumo de sustancia $(28,3 \%)$. Conclusión: el tipo de violencia denunciada con mayor frecuencia fue la violencia intrafamiliar de pareja y afectó a la gente de edad joven y productiva mostrando un comportamiento de género y factores de riesgo social.

Palabras clave: violencia doméstica; agresor; factores de riesgo; violencia de género; trastornos mentales; trastorno por abuso de sustancia.

\begin{abstract}
:
Nowadays, domestic violence is a public health problem due to its magnitude and socio-sanitary effects. Aim: Examine the epidemiological, sociodemographic and psychopathological characteristics of people denounced as aggressors in the city of Córdoba. Material and Methods: exploratory, retrospective and cross-sectional study of people denounced as alleged intrafamiliar violence aggressors at the Family Violence Prosecution Unit of the Córdoba Province Court of Law throughout the period from June 2011 to May 2012. Results: $86 \%$ were men and $14 \%$ women of an age \pm 35 . Couple violence was the most predominant in 69\%. The denounced people had an educational level in $60.7 \%$. The most frequent aggression was threat in $45.6 \%$. Then, serious injuries in $35.3 \%$. Most of the cases did not have a diagnosis of mental disorder (64.3\%). The most common disorder was substance abuse $(28.3 \%)$.

Conclusion: The most frequently denounced form of violence was the intimate partner violence and it affects people at an early and productive age, showing a gender behavior and social risk factors.
\end{abstract}

Keywords: family violence; aggressor; risk markers; gender violence; mental disorder, substance related disorders.

\footnotetext{
${ }^{1}$ Departamento de Salud Mental. Secretaría de Salud Pública y Ambiente Facultad de Ciencias Médicas Universidad Nacional de Córdoba.
} 


\section{Introducción}

La Organización Mundial de la Salud (2002) define la violencia como "el uso intencional de la fuerza física o el poder, ya sea en grado de amenaza o efectivo, contra uno mismo, otra persona $o$ un grupo o comunidad, que cause 0 tenga muchas probabilidades de causar lesiones, muerte, daños psicológicos, trastornos del desarrollo o privaciones"(1). Se clasifica la violencia en tres grandes categorías según el autor del acto violento: violencia dirigida contra uno mismo, violencia interpersonal, dentro de ella se distinguen dos categorías: a) Violencia intrafamiliar 0 doméstica (mayoritariamente ejercida por el hombre hacia la mujer) b) violencia comunitaria: se produce entre individuos no relacionados entre sí, que pueden conocerse 0 no y que generalmente acontece fuera del hogar ${ }^{(1)}$. Violencia colectiva es aquella que se produce por personas que se identifican a sí mismas como miembro de un grupo, frente a otro grupo o conjunto de individuos, con objeto de lograr objetivos políticos, económicos 0 sociales $^{(1)}$.

Se reconoce que la violencia es un problema social complejo y multi-causal considerado uno de los principales problemas de salud pública y para la región de Latinoamérica es una de las principales causas de morbi-mortalidad para los jóvenes ${ }^{(1,2,3)}$. Entre los factores que conllevan a situaciones de violencia la bibliografía identifica a factores biológicos, psicológicos y socioculturales $^{(4,5,6)}$. Respecto a los factores biológicos se reconoce padecer trastornos mentales del espectro impulsivo-agresivo y el consumo de sustancias como el de mayor prevalencia ${ }^{(1)}$. Como factores psicológicos las distorsiones cognitivas, cogniciones particulares sobre la mujer y la relación de la pareja, mal control de la ira e impulsividad, bajo grado de empatía, escasas habilidades comunicacionales, déficit en estrategias para resolver problemas ${ }^{(4-5)}$. Como factores socio-familiares se reconoce a familias mono-parentales, problemas de violencia transgeneracional, maltrato infantil, desempleo, bajo nivel educativo, pobreza, migraciones entre otros $^{(1,5,6,7,8)}$.

Implementar medidas de prevención más específicas. Aún cuando en las últimas décadas se avanzó en el reconocimiento de los factores de riesgo de situaciones de violencia y el desarrollo de medidas de prevención, se reconoce que la falta de estudios es uno de los obstáculos más importantes para conocer el verdadero alcance del problema e implementar medidas de intervención y prevención más específicas $^{(9)}$. De ello surge el objetivo del presente trabajo: explorar las características epidemiológicas sociodemográficas y psicopatológicas de personas denunciadas como agresores en la ciudad de Córdoba.

\section{Material y Método}

Estudio exploratorio, retrospectivo y transversal de los registros de personas denunciadas como presuntos agresores de violencia intrafamiliar en la fiscalía de Violencia Familiar de Tribunales Judiciales Provinciales № 2 de la Provincia de Córdoba Capital en el período comprendido entre junio 2011 a mayo 2012.

Los datos se obtuvieron de los registros de entrevistas clínicas de diagnóstico pericial realizadas por médicos especialistas en psiquiatría a 861 personas denunciadas como presuntos agresores. Como instrumento diagnóstico se utilizó la décima versión de la Clasificación Estadística Internacional de Enfermedades y otros Problemas de Salud de la Organización Mundial de la Salud (CIE 10). Dicho sistema de categorización de las enfermedades asigna denominación de entidades morbosas acorde a criterios preestablecidos ${ }^{(11)}$.

Las variables consideradas en el estudio fueron:

Caracterización socio-demográficas: edad (en años), sexo, nivel educativo (analfabeto, estudios primarios, estudios secundarios, estudios superiores, dato no consignado). Situación laboral (trabaja, no trabaja, dato no consignado). Tipo de delito: privación ilegítima de la libertad, no respeta exclusión, lesiones graves, homicidio, se desconoce, daño, amenazas, abuso sexual.

Tipo de vínculo con la víctima: pareja, expareja, padre/madre, hijo/a, otro vínculo familiar (se agruparon las variables hermano, cuñado, suegro, hijastro, tío, sobrino, ex cuñado, abuelo, primo, yerno, consuegro, nuera, pareja del padre, pareja de la ex pareja, madrasta, ex pareja de la mujer, hijastro, tío, sobrino, ex cuñado, abuelo, primo, yerno, consuegro, nuera, pareja del padre, pareja de la ex pareja, madrasta, ex pareja de la mujer).

Trastorno mentales y del comportamiento
codificados con CIE-10 (F10-19: trastorno mentales y del comportamiento debido a consumo de sustancias, F20-29 esquizofrenia, trastorno esquizotípico y trastornos delirantes, F30-39 trastornos del humor, F40-49 trastornos neuróticos, secundarios a situaciones estresantes y somatomorfos; F50-59 trastornos asociados a disfunciones fisiológicas y a factores somáticos, F60-69 trastornos de la personalidad 
y del comportamiento del adulto, F70-79 retraso mental. El consumo de sustancia se tipificó por tipo de sustancia consumida según CIE_10.

Los datos se exploraron mediante el software IBM Statistical Package for the social Sciences (SPSS v 13.0) y se procesaron por análisis de frecuencia y porcentajes.

\section{Resultados}

Se analizaron 861 registros de pericias psiquiátricas a personas denunciadas como agresores de violencia intrafamiliar en la ciudad de Córdoba, el $86 \%(n=740)$ fueron varones y el $14 \%(n=121)$ mujeres. La media de edad de la muestra fue de 35 años, con una edad mínima de 17 y máxima de 74 años. La media de edad para mujeres fue de 34 años y para los hombres de 36 años. El grupo etario más prevalente fue el de 30 a 39 años con el $35,1 \%(n=302)$ y luego el de 20 a 29 años con el 28,6\% ( $n=246)$. La caracterización socio demográfica de presuntos agresores por edad y género se presentan en la tabla 1.

Tabla 1: Caracterización sociodemográfica de los agresores

\begin{tabular}{|c|c|c|c|c|c|c|}
\hline Edad & femenino & $\%$ & masculino & $\%$ & total & $\%$ \\
\hline$>20$ & 5 & $4,13 \%$ & 28 & $3,78 \%$ & 33 & $3,8 \%$ \\
\hline $20-29$ & 39 & $32,23 \%$ & 207 & $27,97 \%$ & 246 & $28,6 \%$ \\
\hline $30-39$ & 49 & $40,50 \%$ & 253 & $34,19 \%$ & 302 & $35,1 \%$ \\
\hline $40-49$ & 18 & $14,88 \%$ & 147 & $19,86 \%$ & 165 & $19,2 \%$ \\
\hline $50-59$ & 8 & $6,61 \%$ & 67 & $9,05 \%$ & 75 & $8,7 \%$ \\
\hline $60-69$ & 1 & $0,83 \%$ & 24 & $3,24 \%$ & 25 & $2,9 \%$ \\
\hline $\begin{array}{l}\text { 70-mas } \\
\text { sin }\end{array}$ & 1 & $0,83 \%$ & 6 & $0,81 \%$ & 7 & $0,8 \%$ \\
\hline consignar & 0 & $0,00 \%$ & 8 & $1,08 \%$ & 8 & $0,9 \%$ \\
\hline total & 121 & $100,00 \%$ & 740 & $100,00 \%$ & 861 & $100,0 \%$ \\
\hline \multicolumn{7}{|l|}{$\begin{array}{l}\text { Nivel } \\
\text { educativo }\end{array}$} \\
\hline $\begin{array}{l}\text { analfabeto } \\
\text { primario }\end{array}$ & 4 & $3,31 \%$ & 15 & $2,03 \%$ & 19 & $2,2 \%$ \\
\hline $\begin{array}{l}\text { completo } \\
\text { primario }\end{array}$ & 32 & $26,45 \%$ & 184 & $24,86 \%$ & 216 & $25,1 \%$ \\
\hline $\begin{array}{l}\text { incompleto } \\
\text { secundario }\end{array}$ & 2 & $1,65 \%$ & 62 & $8,38 \%$ & 64 & $7,4 \%$ \\
\hline $\begin{array}{l}\text { completo } \\
\text { secundario }\end{array}$ & 24 & $19,83 \%$ & 88 & $11,89 \%$ & 112 & $13,0 \%$ \\
\hline $\begin{array}{c}\text { incompleto } \\
\text { terciario }\end{array}$ & 17 & $14,05 \%$ & 86 & $11,62 \%$ & 103 & $12,0 \%$ \\
\hline $\begin{array}{l}\text { completo } \\
\text { terciario }\end{array}$ & 2 & $1,65 \%$ & 7 & $0,95 \%$ & 9 & $1,0 \%$ \\
\hline $\begin{array}{l}\text { incompleto } \\
\text { universitari }\end{array}$ & 0 & $0,00 \%$ & 1 & $0,14 \%$ & 1 & $0,1 \%$ \\
\hline $\begin{array}{l}\text { o completo } \\
\text { universitari }\end{array}$ & 3 & $2,48 \%$ & 10 & $1,35 \%$ & 13 & $1,5 \%$ \\
\hline $\begin{array}{l}\text { o incompleto } \\
\text { no }\end{array}$ & 1 & $0,83 \%$ & 4 & $0,54 \%$ & 5 & $0,6 \%$ \\
\hline consignado & 36 & $29,75 \%$ & 283 & $38,24 \%$ & 319 & $37,0 \%$ \\
\hline total & 121 & $100,00 \%$ & 740 & $100,00 \%$ & 861 & $100,0 \%$ \\
\hline \multicolumn{7}{|l|}{$\begin{array}{l}\text { Situación } \\
\text { laboral }\end{array}$} \\
\hline trabaja & 52 & $42,98 \%$ & 367 & $49,59 \%$ & 419 & $48,7 \%$ \\
\hline $\begin{array}{l}\text { no trabaja } \\
\text { no }\end{array}$ & 19 & $15,70 \%$ & 33 & $4,46 \%$ & 52 & $6,0 \%$ \\
\hline consignado & 50 & $41,32 \%$ & 340 & $45,95 \%$ & 390 & $45,3 \%$ \\
\hline total & 121 & $100,00 \%$ & 740 & $100,00 \%$ & 861 & $100,0 \%$ \\
\hline
\end{tabular}

\begin{tabular}{lcccccc|} 
oficio & 35 & $67,31 \%$ & 254 & $69,21 \%$ & 289 & $69,0 \%$ \\
otro & 13 & $25,00 \%$ & 99 & $26,98 \%$ & 112 & $26,7 \%$ \\
profesional & 4 & $7,69 \%$ & 14 & $3,81 \%$ & 18 & $4,3 \%$ \\
total & 52 & $100,00 \%$ & 367 & $100,00 \%$ & 419 & $100,0 \%$ \\
\hline
\end{tabular}

Al explorar el nivel educativo se encontró que el $60,7 \%(n=523)$ de los casos tenía nivel educativo, en el $37 \%(n=319)$ no estaba consignado el dato del nivel educativo y el $2,2 \% \quad(n=19)$ era analfabeto. El grupo con nivel educativo tenía el $25,1 \%(n=216)$ nivel primario completo, el $13 \%$ $(n=112)$ nivel secundario completo, el $1 \%(n=9)$ terciario completo y el $1,5 \%(n=13)$ universitario completo. El análisis del nivel educativo por género se presenta en la tabla 1.

En relación a la situación laboral al momento de la denuncia el $54,7 \%(n=471)$ de los casos tenía consignado el dato (el $48,7 \%$ tenía trabajo y el $2,2 \%$ no tenía trabajo) y en el $45,3 \%(n=390)$ no estaba consignado el dato. El $69 \%$ trabajaba en oficios (como albañil, pintor, plomero, carpintero), otro trabajo el $26,7 \%$ (empleado, operario) y el $4,3 \%$ profesionales. El análisis de la situación laboral por genero mostró que el $42,98 \%$ de las mujeres trabajaban (la mayor parte de ellas en oficios $67,3 \%$, otro trabajo $25 \%$ y profesionales 7,6\%). El análisis de los resultados de la situación laboral se muestra en la tabla 1.

Respecto al tipo de lesión que motivó la denuncia las más frecuentes fue la amenaza en el $45,6 \%$ $(n=393)$, luego lesiones graves $35,3 \%(n=304)$ y no respeta la exclusión $7,7 \%(n=66)$. El análisis del tipo de lesión por género mostró que el homicidio se encontró con una mayor prevalencia en mujeres con el 5,67\% (n=7) (P >0,008) mostrando diferencias significativas. Los resultados analizados por tipo de lesión y género se presentan en tabla 2.

En relación al tipo de violencia según el vínculo con la persona agredida se observó que la violencia de pareja fue la más frecuente en el $69 \%(n=599)$ de los casos (en el $57 \%$ fue la pareja y en el $12 \%$ fue la ex pareja). Luego, en el $18,4 \%(n=158)$ la agresión fue por otro familiar, en el $12 \% \quad(n=104)$ fue por hijo (violencia filioparental; agresión de hijos contra padres) y en el $5,3 \% \quad(n=46)$ fue el padre. Los resultados se presentan en tabla 2.

Tabla 2: Caracterización por tipo de lesión y vinculo

\begin{tabular}{|lcccccc|}
\hline $\begin{array}{l}\text { tipo de } \\
\text { lesión }\end{array}$ & femenino & $\%$ & masculino & $\%$ & total & $\%$ \\
\hline $\begin{array}{l}\text { abandono } \\
\text { abuso }\end{array}$ & 0 & $0,00 \%$ & 1 & $0,14 \%$ & 1 & $0,1 \%$ \\
$\begin{array}{l}\text { sexual } \\
\text { amenazas }\end{array}$ & 0 & $0,00 \%$ & 7 & $0,95 \%$ & 7 & $0,8 \%$ \\
daño & 60 & $49,59 \%$ & 333 & $45,00 \%$ & 393 & $45,6 \%$ \\
violación & 0 & $4,96 \%$ & 16 & $2,16 \%$ & 22 & $2,6 \%$ \\
\end{tabular}


ARTÍCULO ORIGINAL

\begin{tabular}{|c|c|c|c|c|c|c|}
\hline homicidio & 7 & $5,79 \%$ & 8 & $1,08 \%$ & 15 & $1,7 \%$ \\
\hline $\begin{array}{l}\text { lesiones } \\
\text { leves }\end{array}$ & 1 & $0,83 \%$ & 16 & $2,16 \%$ & 17 & $2,0 \%$ \\
\hline $\begin{array}{l}\text { lesiones } \\
\text { graves }\end{array}$ & 38 & $31,40 \%$ & 266 & $35,95 \%$ & 304 & $35,3 \%$ \\
\hline $\begin{array}{l}\text { no respeta } \\
\text { exclusión } \\
\text { privación }\end{array}$ & 5 & $4,13 \%$ & 61 & $8,24 \%$ & 66 & $7,7 \%$ \\
\hline $\begin{array}{l}\text { ilegitima de } \\
\text { la libertad }\end{array}$ & 0 & $0,00 \%$ & 13 & $1,76 \%$ & 13 & $1,5 \%$ \\
\hline robo & 1 & $0,83 \%$ & 1 & $0,14 \%$ & 2 & $0,2 \%$ \\
\hline $\begin{array}{c}\text { tentativa } \\
\text { de homicidio }\end{array}$ & 0 & $0,00 \%$ & 2 & $0,27 \%$ & 2 & $0,2 \%$ \\
\hline $\begin{array}{l}\text { no } \\
\text { consignado }\end{array}$ & 3 & $2,48 \%$ & 15 & $2,03 \%$ & 18 & $2,1 \%$ \\
\hline total & 121 & $100,00 \%$ & 740 & $100,00 \%$ & 861 & $100,0 \%$ \\
\hline \multicolumn{7}{|l|}{$\begin{array}{l}\text { relación con } \\
\text { la victima }\end{array}$} \\
\hline pareja & 55 & $45,45 \%$ & 441 & $59,59 \%$ & 496 & $57,6 \%$ \\
\hline expareja & 10 & $8,26 \%$ & 93 & $12,57 \%$ & 103 & $12,0 \%$ \\
\hline padre & 9 & $7,44 \%$ & 37 & $5,00 \%$ & 46 & $5,3 \%$ \\
\hline $\begin{array}{l}\text { hijo } \\
\text { otro }\end{array}$ & 24 & $19,83 \%$ & 34 & $4,59 \%$ & 58 & $6,7 \%$ \\
\hline $\begin{array}{l}\text { vinculo } \\
\text { familiar }\end{array}$ & 23 & $19,01 \%$ & 135 & $18,24 \%$ & 158 & $18,4 \%$ \\
\hline total & 121 & $100,00 \%$ & 740 & $100,00 \%$ & 861 & $100,0 \%$ \\
\hline
\end{tabular}

Al explorar la presencia de trastornos mentales se observó que el $64,3 \%(n=554)$ de los casos no presentaban diagnóstico de trastorno mental y en el 35,4\% $(n=307)$ se encontró un diagnóstico de trastorno mental. El $37 \% \quad(n=274)$ fueron hombres y el $27 \%(n=33)$ mujeres. Respecto a los trastornos mentales se encontró que el más frecuente fue el consumo de sustancias $28,3 \%$ $(n=244)$ para ambos sexos. En el $51,2 \%$ se detectó policonsumo y en el $41 \%$ el consumo de alcohol. Otros trastornos mentales detectados fueron depresión en el 2,3\% $(n=20)$ con mayor prevalencia en mujeres, trastorno bipolar en el $0,3 \%(n=3)$, trastornos psicóticos en el 1,7\% $(n=15)$ y esquizofrenia, en el $1,6 \% \quad(n=14)$ trastornos de la personalidad y en el $0,5 \%(n=4)$ retraso mental. El análisis de los resultados por trastorno mental y género se consigna en la tabla 3.

Tabla 3: Caracterizacion por trastorno mental y tipo de consumo de sustancia

\begin{tabular}{|lcccccc|}
\hline psicopatología & femenino & $\%$ & masculino & $\%$ & total & $\%$ \\
\hline $\begin{array}{l}\text { no presenta } \\
\text { trastorno }\end{array}$ & 88 & $72,73 \%$ & 466 & $62,97 \%$ & 554 & $64,3 \%$ \\
$\begin{array}{l}\text { bipolar } \\
\quad \text { depresión }\end{array}$ & 0 & $0,00 \%$ & 3 & $0,41 \%$ & 3 & $0,3 \%$ \\
$\begin{array}{l}\text { esquizofreni } \\
\text { a, trastorno }\end{array}$ & 6 & $4,96 \%$ & 14 & $1,89 \%$ & 20 & $2,3 \%$ \\
$\begin{array}{l}\text { esquizotipico, } \\
\text { trastorno }\end{array}$ & 4 & $3,31 \%$ & 11 & $1,49 \%$ & 15 & $1,7 \%$ \\
$\begin{array}{l}\text { delirante } \\
\text { debilidad }\end{array}$ & 0 & $0,00 \%$ & 4 & $0,54 \%$ & 4 & $0,5 \%$ \\
$\begin{array}{l}\text { mental } \\
\text { trastorno por }\end{array}$ & 0 & $0,00 \%$ & 2 & $0,27 \%$ & 2 & $0,2 \%$ \\
$\begin{array}{l}\text { control de los } \\
\text { impulsos } \\
\quad \begin{array}{l}\text { trastorno de } \\
\text { la personalidad }\end{array}\end{array}$ & 6 & $4,96 \%$ & 8 & $1,08 \%$ & 14 & $1,6 \%$ \\
\hline
\end{tabular}

\begin{tabular}{|lcccccc|}
$\begin{array}{l}\text { síndrome } \\
\text { cerebral } \\
\text { orgánico } \\
\text { trastorno por }\end{array}$ & 1 & $0,83 \%$ & 4 & $0,54 \%$ & 5 & $0,6 \%$ \\
$\begin{array}{l}\text { consumo de } \\
\text { sustancia } \\
\text { total }\end{array}$ & 16 & $13,22 \%$ & 228 & $30,81 \%$ & 244 & $28,3 \%$ \\
\hline $\begin{array}{l}\text { tipo de } \\
\text { consumo }\end{array}$ & 121 & $100,00 \%$ & 740 & $100,00 \%$ & 861 & $100,0 \%$ \\
\hline $\begin{array}{l}\text { alcohol } \\
\text { cocaína }\end{array}$ & 6 & $37,50 \%$ & 94 & $41,23 \%$ & 100 & $41,0 \%$ \\
$\quad$ marihuana & 1 & $0,00 \%$ & 11 & $4,82 \%$ & 11 & $4,5 \%$ \\
$\quad$ policonsum & 9 & $5,25 \%$ & 7 & $3,07 \%$ & 8 & $3,3 \%$ \\
o & $56,25 \%$ & 116 & $50,88 \%$ & 125 & $51,2 \%$ \\
total & 16 & $100,00 \%$ & 228 & $100,00 \%$ & 244 & $100,0 \%$ \\
\hline
\end{tabular}

\section{Discusión}

De los datos obtenidos se observa que el tipo de violencia denunciada con mayor frecuencia fue la violencia doméstica o intrafamiliar de pareja y mostró un comportamiento diferenciado por género ya que los agresores fueron con mayor frecuencia los varones coincidiendo con lo referido en los estudios ${ }^{(6,8,12)}$. Un dato a mencionar es la mayor prevalencia de personas denunciadas como agresores mujeres cuando el tipo de lesión fue homicidio y este aspecto se debería profundizar en su exploración. Los estudios refieren que el $60 \%$ de los países no tienen datos sobre homicidios ${ }^{(12,14)}$.

Se conoce que la violencia ocasiona muertes y consecuencias no mortales de diferente gravedad comprometiendo a los grupos poblacionales más vulnerables, mujeres, niños y personas de tercera edad. Los diferentes tipos de violencia comparten muchos factores de riesgo entre los que se reconoce la edad, desigualdad económica, el consumo de sustancias (alcohol particularmente), antecedentes de maltrato infantil y violencia juvenil $^{(13,14,15,16)}$.

El análisis de los factores socio-demográficos en este estudio mostró como los aspectos más importantes que el grupo etario más comprometido fue el de los 20 a 39 años, el nivel de educación primaría y una situación laboral precaria. La OMS indica como una medida de prevención y promoción de la violencia, que está siendo aplicada en algunos países, ofrecer incentivos a las personas jóvenes en riesgo de violencia para que completen el nivel de educación secundario ${ }^{(14)}$.

Al explorar los trastornos mentales y coincidiendo con lo referido en la bibliografía se observó una baja prevalencia de estos y un comportamiento diferenciado por género observando que el más frecuente en los hombre fue el consumo de sustancias y en las mujeres también consumo de sustancias, depresión y trastornos de personalidad $^{(18,19)}$. Otro aspecto mencionado para la prevención de la violencia (OMS, 2014) son las 
medidas de prevención del consumo de sustancias y sostiene, que si bien "la mayor parte de los países afirman ocuparse del consumo nocivo de alcohol, no obstante el consumo de esta bebida en los países de ingresos más bajos continua siendo elevada"(14). Los resultados obtenidos en este estudio mostraron una elevada prevalencia de consumo de sustancia (policonsumo y consumo de alcohol) tanto en varones como en mujeres. El consumo de sustancias también ha sido identificado como un factor de riesgo de repetir nuevos episodios de violencia ${ }^{(3,}$ $7,9,11,16,17)$

La prevención y el abordaje de la violencia es un problema social complejo que requiere del estudio y la colaboración de las área salud, justicia, seguridad ciudadana y es indispensable la articulación y el trabajo colaborativo intersectorial para su prevención.

Para reducir los diferentes tipos de violencia la OMS sugiere que es indispensable contar con información de lo que sucede y el déficit de información de la magnitud del problema se da en todos los sectores ${ }^{(14)}$. Es indispensable contar con información que contribuya a diseñar líneas de prevención a nivel individual, relacional y comunitario, que promuevan vínculos de respeto y no violencia, que se reduzca el consumo de sustancias y se promueva la igualdad de género ${ }^{(14)}$.

De lo explorado podemos concluir que el tipo de violencia denunciada con mayor frecuencia fue la violencia intrafamiliar y dentro de ella la de pareja, que afecta a la gente de edad joven y productiva y mostró un comportamiento diferenciado por género. Se detectaron, en las personas denunciadas, factores de riesgo social, como nivel de educación, situación laboral y consumo de sustancias, todos estos aspectos sociosanitarios que requieren de acciones y medidas de prevención intersectoriales.

\section{Bibliografía}

1. Informe mundial sobre la violencia y la salud. Washington, D.C. Organización Panamericana de la Salud, Oficina Regional para las Américas de la Organización Mundial de la Salud, 2003.Disponible en: http://apps.who.int/iris/bitstream/10665/ 112670/1/9275315884_spa.pdf

2. Serfaty E, Foglia L, Masautis A, Negri G. Mortalidad por causas violentas en adolescentes y jóvenes de 10 a 24 años. Vertex. 2007; (40): 25-30.

3. Cardona D, Peláez E, Aidar T, Ribotta B, Alvarez MF. Mortalidad por causas externas en tres ciudades latinoamericanas: Córdoba (Argentina), Campinas (Brasil) y Medellín (Colombia), 1980-2005. R. Bras. Est. Pop., São Paul. 2008; 25 (2) 335-352.

4. Enrique Echeburúa, Paz de Corral, Javier FernándezMontalvo y Pedro J. Amor. ¿Se puede y se debe tratar psicológicamente a los hombres violentos contra la pareja? Papeles del Psicólogo Mayo, N॰88,2004

5. Enrique Echeburúa y Pedro Amor. Perfil psicopatológico e intervención terapéutica con agresores contra la pareja. Revista Española de Medicina Legal. 2010; 36(3):117-121.

6. Pastor Bravo M., Rodes Loret F., Navarro Escayola E. Perfil del agresor en la violencia de género. Boletín Galego de Medicina Legal e Forense №. 16. Diciembre 2009.

7. Echeburua Odriozola y P. de Corral E. Violencia doméstica: ¿es el agresor un enfermo? FMC 2004;11(6):297-303.

8. M. Castellano Arroyo, E. Lachica López, A. Molina Rodríguez3 y $\mathrm{H}$. Villanueva de la Torre. Violencia contra la mujer. El perfil del agresor: criterios de valoración del riesgo. Cuadernos de Medicina Forense № 35 - Enero 2004.

9. F. Caravaca Sánchez, M. García-Jarillo, C. SánchezAlcaraz Martínez, A. Luna Maldonado. Estudio del consumo de sustancias psicoactivas y conflictividad familiar en sujetos condenados por delitos de violencia de género. Cuadernos Medico Forense 2014;20(23):92-98.

10. A. Andrés Pueyo, S. López, y E. Álvarez. Valoración del riesgo de violencia contra la pareja por medio de la SARA. Papeles del Psicólogo, 2008. Vol. 29(1), pp. 107-122.

11. Clasificación Estadística Internacional de Enfermedades y Problemas Relacionados con la Salud (Cie 10), edición 2008 http://publications.paho.org/product.phpproductid=990\& cat $=85$ \& page $=1 \% 20$

12. Pedro J. Amor. Variables psicosociales y riesgo de violencia grave en parejas con abuso de sustancias toxicas y maltrato previo. Acción psicológica,junio2012. vol.9,n.1.3-18.ISSN:1578-9008X.

13. D'Angelo $L$, Hubez G(), De Cesare MD, Farace $R$, Ricaurte HI. Estudio Nacional sobre Violencias contra las mujeres. Informe preliminar basado en la International Violence Against Women Survey. Disponible en: http://www.jus.gob.ar/media/3128472/Encuesta\%20de \%20violencias\%20contra\%20las\%20mujeres\%20\%20F INAL\% 2026 - 10.pdf

14. OMS. Informe sobre la situación mundial de la prevención de la violencia 2014. Disponible en: http://apps. who.int/iris/bitstream/10665/145089/1/WHO NMH_NVI14.

15. Chérrez-Bermejo, $R$ Alás-Brun. Consumo de sustancias y trastornos de salud mental en agresores de violencia de género ingresados en prisión. Un estudio descriptivo. Revista Española de Sanidad Penitenciaria 2014; 16: 29-37

16. Denuncias de Violencia familiar. Informe estadístico elaborado por el centro de estudios y proyectos judiciales -Tribunal Superior de Justicia. Disponible en: http://www.justiciacordoba.gob.ar/cepj/_Estudios/Estudi osDetalle.aspxidCategoria=1 \&idSubCategoria $=7$

17. Alicia Ballester y Lidón Villanueva. Valoración psicológica en delitos de violencia de género mediante el Inventario Clínico y Multiaxial de Millon III (MCMI-III). Anuario de Psicología Jurídica 201424 (2014) 9-18

18. Deborah M. Capaldi, Naomi B. Knoble, Joann Wu Shortt, and Hyoun K. Kim. A Systematic Review of Risk Factors for Intimate Partner Violence Partner Abuse. 2012 April; 3(2): 231-280.

19. Fals-Stewart W1, Kennedy C. Addressing intimate partner violence in substance-abuse treatment. J Subst Abuse Treat. 2005 Jul; 29(1):5-17. 